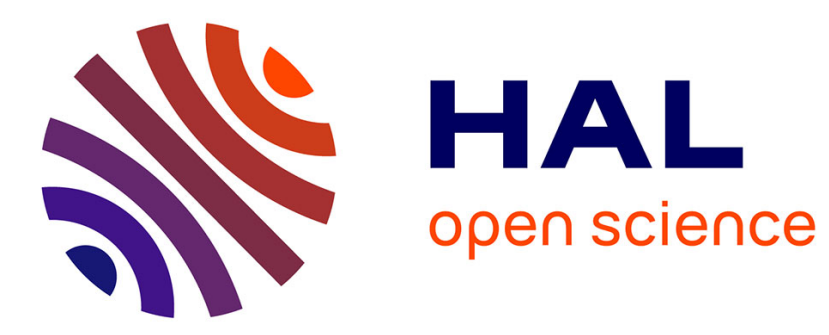

\title{
Analysis of non-steady-state distribution functions for grain growth and coarsening
}

Franz Dieter Fischer, Jiri Svoboda, Ernst Gamsjäger

\section{To cite this version:}

Franz Dieter Fischer, Jiri Svoboda, Ernst Gamsjäger. Analysis of non-steady-state distribution functions for grain growth and coarsening. Philosophical Magazine, 2009, 89 (17), pp.1425-1438. 10.1080/14786430902988757 . hal-00514026

\section{HAL Id: hal-00514026 \\ https://hal.science/hal-00514026}

Submitted on 1 Sep 2010

HAL is a multi-disciplinary open access archive for the deposit and dissemination of scientific research documents, whether they are published or not. The documents may come from teaching and research institutions in France or abroad, or from public or private research centers.
L'archive ouverte pluridisciplinaire HAL, est destinée au dépôt et à la diffusion de documents scientifiques de niveau recherche, publiés ou non, émanant des établissements d'enseignement et de recherche français ou étrangers, des laboratoires publics ou privés. 


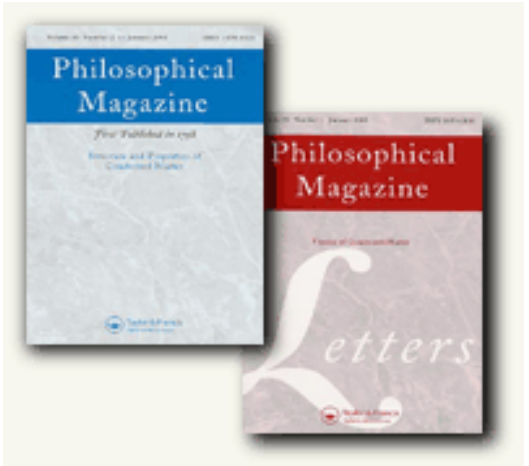

\section{Analysis of non-steady-state distribution functions for grain growth and coarsening}

\begin{tabular}{|r|l|}
\hline Journal: & Philosophical Magazine \& Philosophical Magazine Letters \\
\hline Manuscript ID: & TPHM-09-Jan-0041.R1 \\
\hline Journal Selection: & Philosophical Magazine \\
\hline $\begin{array}{r}\text { Date Submitted by the } \\
\text { Author: }\end{array}$ & 22-Apr-2009 \\
\hline Complete List of Authors: & $\begin{array}{l}\text { Fischer, Franz Dieter; Montanuniversität Leoben, Institute of } \\
\text { Mechanics } \\
\text { Svoboda, Jiri; Academy of Sciences of the Czech Republic, Institute } \\
\text { of Physics of Materials } \\
\text { Gamsjäger, Ernst; Montanuniversität Leoben, Institute of Mechanics }\end{array}$ \\
\hline Keywords: & coarsening, grain growth, thermodynamics \\
\hline Keywords (user supplied): & modeling, distribution concept \\
\hline \multicolumn{2}{|l}{}
\end{tabular}

\section{$\diamond$ ScholarONE \\ Manuscript Central}




\title{
Analysis of non-steady-state distribution functions for grain growth and coarsening
}

\author{
F.D. Fischer ${ }^{*}$, J. Svoboda $^{2}$, E. Gamsjäger ${ }^{1}$, \\ ${ }^{1}$ Institute of Mechanics, Montanuniversität Leoben, \\ Franz-Josef-Straße 18, A-8700 Leoben, Austria \\ ${ }^{2}$ Institute of Physics of Materials, Academy of Sciences of the Czech Republic, \\ Žižkova 22, CZ-616 62, Brno, Czech Republic
}

\begin{abstract}
The description of non-steady-state grain growth or precipitate coarsening by means of object radius distribution functions with multiple time dependent parameters (distribution concept) seems to be promising. The present paper deals with the simplest case of non-steady-state distribution functions with two parameters - the first one scaling the object radius, the second one determining the shape of the distribution function. The main question concerns the physical basis behind the evolution of these two parameters. The principle of maximum dissipation has proven to be a proper tool to derive the evolution equations. Semi-analytical solutions for the evolving parameters of arbitrary two-parameter distribution functions can be developed. As examples Kirkaldy and Weibull-type distribution functions are investigated. It is shown that the parameters of the Kirkaldy distribution function are not independent, and, thus, the general non-steady-state analysis fails. For a Weibull-type distribution function nearly exact and simple analytical expressions for both parameters are presented and discussed for the grain growth and coarsening cases.
\end{abstract}

Keywords: Grain growth; Coarsening; Thermodynamics; Modeling; Distribution concept

* corresponding author Mechanik@mu-leoben.at 


\section{Introduction}

Non-steady-state distributions of grains are a topic of current research. These distributions have been constructed by the multi phase-field (MPF) model, the Monte Carlo Potts (MCP) model and the model based on principle of maximum dissipation (PMD) in recent studies.

A set of continuous field variables $\phi_{i}(\underline{r}, t)$, depending on the position $\underline{r}$ and time $t$ is defined within the MPF model. These field variables $\phi_{i}$, used as order parameters, overtake the role of the volume fraction of a distinct orientation. A free energy functional must now be proposed including additional weighted gradient terms of the set of $\phi_{i}$. The MPF model involves a remarkable set of de-facto unknown parameters, for details see e.g. [1]. A computationally improved version was recently presented in [2] and applied to simulations of normal grain growth reflecting the Hillert theory.

Zöllner and Streitenberger [3] used the MCP model, which is computationally very efficient and compares the energy state of a lattice site with a possible neighbouring state. Then a probability is defined for a flip to this neighbouring state. This concept needs the definition of the energy states as functions of the orientation of a lattice site and its neighbours. In addition to the physical parameters (e.g. the grain boundary energy) several technical parameters must be set properly. Then is possible to reflect the Hillert theory of normal grain growth.

Of course, a significant number of parameters appears again. A proper selection allows also reflecting the Hillert theory for normal grain growth.

The PMD concept is based on the proper formulation of the total dissipation $Q$ and of the total Gibbs energy $G$ of the system both in the case of grain growth and coarsening, see [4]. The Gibbs energy $G$ contains the total grain boundary (or interface) energy, which unlike the other contributions to the total Gibbs energy $G$, may change with time. Svoboda et al. could show in [5] how this concept can be applied to modelling of non-steady grain growth. Two possible approaches were introduced. The evolution equations for the radii of individual grains were obtained by using the multigrain concept and for the parameters of the distribution functions by means of the distribution concept. The latter concept has been demonstrated for Rayleigh's 
distribution [6] with the mean grain size as parameter. The authors have shown in [7] how the distribution concept can be used to derive the evolution equation for the grain radius scaling parameter $a(t)$ as well as for the radius $R(t)$ of the individual grains for several prominent one-parametric distribution functions. The value of the critical radius $R_{c}$ follows then automatically from the condition $\dot{R}\left(R_{c}\right)=0$.

The distribution concept is also supported by very recent automated analysis methods for three-dimensional polycrystalline microstructures as presented by Groeber et al. $[8,9]$. This gives a strong motivation to look deeper into the analysis of distribution functions with multiple parameters.

In this paper a non-steady-state distribution function is investigated with an object radius $R_{c}(t)$, and a dimension-free parameter $\beta(t)$, determining the shape of the distribution function, for both the grain growth and coarsening case. A general derivation of the two equations for $a(t)$ and $\beta(t)$ is given and applied to the Weibull-type distribution function, see e.g. [7], with the special case of the Rayleigh/Louat distribution function, see e.g. [6]. In this context it is worth to note that the case with $a(t)$ and $\beta=$ const is correctly named as self-similar state, but many authors use the denomination "steady-state" for this case, see also [5]. Consequently, we use for $a(t)$ and $\beta(t)$ the denomination "non-steady-state". Finally, the authors would like to remark that a spherical approximation of equiaxed grains is a somewhat coarse approximation. Refined three-dimensional vertex models are available and summarized in a very recent paper [10]. However this approach is extremely complicated. Our study is concentrated on the grain size distribution functions with a low number of time dependent parameter, which allows a simple, effective and well established description with the average grain radius as a specific parameter.

\section{Theory}

\subsection{Description of the distribution function}


The distribution concept, outlined in detail in [5], allows describing the grain growth by means of the evolution of the grain-radius distribution function. The evolution of the distribution function $f\left(R, a_{1}(t), \ldots, a_{n}(t)\right)$ is based on the evolution of the parameters $a_{1}(t), \ldots, a_{n}(t)$, the evolution equations of which can be derived from the Onsager thermodynamic extremal principle. Let us deal in detail with two-parametric distribution functions, in which the first parameter $a$ is used for the scaling of the object radius $R$ and the second parameter $\beta$ influences the shape of the distribution function for the normalized coordinate $r=R / a$. Then one can write the distribution function in the form $f(r, a(t), \beta(t))$. As the distribution function must meet the normalization condition for the constant total volume of the objects

$$
\int_{0}^{\infty} R^{3} f d R=a^{4} \int_{0}^{\infty} r^{3} f d r=1,
$$

the distribution function can be written without any loss of generality as

$$
f(r, a, \beta)=\frac{1}{a^{4}} h(r, \beta) .
$$

\subsection{Derivation of the evolution equations}

\subsubsection{Classification into two cases}

We distinguish two cases, the growth case and the coarsening case, which are characterized by a Gibbs energy parameter $A$ and a dissipation multiplier $B(R)$ as outlined already in [4]:

Grain boundary migration controlled grain growth is considered in the growth case. Then the parameter $A$ is set to $2 \pi \gamma$ with $\gamma$ being the specific grain boundary energy. The function $B(R)$ is defined as $2 \pi R^{2} / M$ with $M$ being the grain boundary mobility. 


\subsubsection{Formulation of the total Gibbs energy $G$ and its derivatives with respect to $a$ and $\beta$}

The Gibbs energy $G$ of the system can be taken from [5] sect. 3 as

$$
G=A \int_{0}^{\infty} R^{2} f d R=A a^{3} \int_{0}^{\infty} r^{2} f d r=\frac{A}{a} \int_{0}^{\infty} r^{2} h(r, \beta) d r .
$$

Using the chain rule for calculation of partial derivatives one obtains

$$
\frac{\partial f}{\partial a}=-\frac{1}{a^{5}}\left(4 h+r \frac{\partial h}{\partial r}\right)
$$

Then using Eq. (3) and applying the integration per parts with $r^{3} h=0$ for $r=0$ or $r \rightarrow \infty$ one obtains

$$
\frac{\partial G}{\partial a}=-\frac{2^{\ell+1} \pi \gamma}{a^{2}} \int_{0}^{\infty} r^{2} h d r
$$

furthermore

$$
\frac{\partial G}{\partial \beta}=\frac{2^{\ell+1} \pi \gamma}{a} \int_{0}^{\infty} r^{2} \frac{\partial h}{\partial \beta} d r
$$


2.2.3. Formulation of the dissipation $Q$ and its second derivatives with respect to the rates $\dot{a}$ and $\dot{\beta}$

The dissipation $Q$ can be taken from [5], sect. 3. as

$$
\begin{aligned}
& Q=\int_{0}^{\infty} \frac{B(R)}{f}\left(\dot{a} \int_{R}^{\infty} \frac{\partial f}{\partial a} d R^{\prime}+\dot{\beta} \int_{R}^{\infty} \frac{\partial f}{\partial \beta} d R^{\prime}\right)^{2} d R= \\
& 2^{\ell+1} \pi b_{\ell} a^{\ell+5} \int_{0}^{\infty} \frac{r^{\ell+2}}{f}\left(\dot{a} \int_{r}^{\infty} \frac{\partial f}{\partial a} d r^{\prime}+\dot{\beta} \int_{r}^{\infty} \frac{\partial f}{\partial \beta} d r^{\prime}\right)^{2} d r= \\
& 2^{\ell+1} \pi b_{\ell} a^{\ell-1} \int_{0}^{\infty} \frac{r^{\ell+2}}{h}\left(-\dot{a} \int_{r}^{\infty}\left(4 h+r \frac{\partial h}{\partial r}\right) d r^{\prime}+\dot{\beta} \int_{r}^{\infty} a \frac{\partial h}{\partial \beta} d r^{\prime}\right)^{2} d r .
\end{aligned}
$$

In the calculations above we used Eqs. (3) and (5).

The second partial derivatives then read

$$
\begin{aligned}
& \frac{1}{2} \frac{\partial^{2} Q}{\partial \dot{a}^{2}}=2^{\ell+1} \pi b_{\ell} a^{\ell-1} \int_{0}^{\infty} \frac{r^{\ell+2}}{h}\left(\int_{r}^{\infty}\left(4 h+r \frac{\partial h}{\partial r}\right) d r^{\prime}\right)^{2} d r, \\
& \frac{1}{2} \frac{\partial^{2} Q}{\partial \dot{\beta}^{2}}=2^{\ell+1} \pi b_{\ell} a^{\ell+1} \int_{0}^{\infty} \frac{r^{\ell+2}}{h}\left(\int_{r}^{\infty} \frac{\partial h}{\partial \beta} d r^{\prime}\right)^{2} d r, \\
& \frac{1}{2} \frac{\partial^{2} Q}{\partial \dot{a} \partial \dot{\beta}}=-2^{\ell+1} \pi b_{\ell} a^{\ell} \int_{0}^{\infty} \frac{r^{\ell+2}}{h}\left(\int_{r}^{\infty}\left(4 h+r \frac{\partial h}{\partial r}\right) d r^{\prime} \cdot \int_{r}^{\infty} \frac{\partial h}{\partial \beta} d r^{\prime}\right) d r .
\end{aligned}
$$

\subsubsection{Evolution equations for $\dot{a}$ and $\dot{\beta}$}

The system of evolution equations is also explained in [5], sect. 3, and writes

$$
\frac{1}{2} \frac{\partial^{2} Q}{\partial \dot{a}^{2}} \dot{a}+\frac{1}{2} \frac{\partial^{2} Q}{\partial \dot{a} \partial \dot{\beta}} \dot{\beta}=-\frac{\partial G}{\partial a}, \quad \frac{1}{2} \frac{\partial^{2} Q}{\partial \dot{a} \partial \dot{\beta}} \dot{a}+\frac{1}{2} \frac{\partial^{2} Q}{\partial \dot{\beta}^{2}} \dot{\beta}=-\frac{\partial G}{\partial \beta} .
$$

We introduce the following abbreviations

$$
\begin{aligned}
& I_{G a}(\beta)=\int_{0}^{\infty} r^{2} h d r, \\
& I_{G \beta}(\beta)=\int_{0}^{\infty} r^{2} \frac{\partial h}{\partial \beta} d r \equiv \frac{d}{d \beta} I_{G a}(\beta),
\end{aligned}
$$




$$
\begin{aligned}
& I_{Q a a}(\beta)=\int_{0}^{\infty} \frac{r^{\ell+2}}{h}\left(3 \int_{r}^{\infty} h d r^{\prime}-r h\right)^{2} d r, \\
& I_{Q \beta \beta}(\beta)=\int_{0}^{\infty} \frac{r^{\ell+2}}{h}\left(\int_{r}^{\infty} \frac{\partial h}{\partial \beta} d r^{\prime}\right)^{2} d r, \\
& I_{Q a \beta}(\beta)=\int_{0}^{\infty} \frac{r^{\ell+2}}{h}\left(\left(3 \int_{r}^{\infty} h d r^{\prime}-r h\right) \cdot \int_{r}^{\infty} \frac{\partial h}{\partial \beta} d r^{\prime}\right) d r .
\end{aligned}
$$

Integration per parts is used in integrals (13c) and (13e). Then the evolution of the system is given by the set of equations

$$
\begin{aligned}
& 2^{\ell+1} \pi b_{\ell} a^{\ell-1} I_{Q a a}(\beta) \dot{a}-2^{\ell+1} \pi b_{\ell} a^{\ell} I_{Q a \beta}(\beta) \dot{\beta}=\frac{2^{\ell+1} \pi \gamma}{a^{2}} I_{G a}(\beta), \\
& -2^{\ell+1} \pi b_{\ell} a^{\ell} I_{Q a \beta}(\beta) \dot{a}+2^{\ell+1} \pi b_{\ell} a^{\ell+1} I_{Q \beta \beta}(\beta) \dot{\beta}=-\frac{2^{\ell+1} \pi \gamma}{a} I_{G \beta}(\beta)
\end{aligned}
$$

and after their simplification as

$$
\begin{aligned}
& I_{Q a a}(\beta) \frac{\dot{a}}{a}-I_{Q a \beta}(\beta) \dot{\beta}=\frac{\gamma}{a^{\ell+2} b_{\ell}} I_{G a}(\beta), \\
& I_{Q a \beta}(\beta) \frac{\dot{a}}{a}-I_{Q \beta \beta}(\beta) \dot{\beta}=\frac{\gamma}{a^{\ell+2} b_{\ell}} I_{G \beta}(\beta) .
\end{aligned}
$$

\section{Solutions}

\subsection{Solution of the evolution equations}

The solution of the set of equations (16), (17) with respect to $\dot{a} / a$ and $\dot{\beta}$ is given by

$$
\begin{aligned}
& \frac{\dot{a}}{a}=\frac{\gamma}{a^{\ell+2} b_{\ell}} \frac{I_{G \beta}(\beta) I_{Q a \beta}(\beta)-I_{G a}(\beta) I_{Q \beta \beta}(\beta)}{D}=\frac{\gamma}{a^{\ell+2} b_{\ell}} F_{a}(\beta), \\
& \dot{\beta}=\frac{\gamma}{a^{\ell+2} b_{\ell}} \frac{I_{G \beta}(\beta) I_{Q a a}(\beta)-I_{G a}(\beta) I_{Q a \beta}(\beta)}{D}=\frac{\gamma}{a^{\ell+2} b_{\ell}} F_{\beta}(\beta)
\end{aligned}
$$

with the determinant $D$ as

$$
D=I_{Q a a}(\beta) I_{Q \beta \beta}(\beta)-\left(I_{Q a \beta}(\beta)\right)^{2}>0
$$


After dividing Eq. (18) by Eq. (19) one obtains

$$
\frac{\dot{a}}{a}=\frac{F_{a}(\beta)}{F_{\beta}(\beta)} \dot{\beta}
$$

Equation (21) can be integrated in time with the result

$$
a=a_{0} \exp \left[\int_{\beta_{0}}^{\beta} \frac{F_{a}(\beta)}{F_{\beta}(\beta)} d \beta^{\prime}\right]=a_{0} \exp \left[J\left(\beta ; \beta_{0}, \ell\right)\right] .
$$

The quantities $a_{0}$ and $\beta_{0}$ represent the initial values of $a$ and $\beta$ at the time $t=0$.

By inserting of Eq. (22) into Eq. (19) one finds the differential equation for $\beta$ as

$$
\dot{\beta}=\frac{\gamma}{a_{0}^{\ell+2} b_{\ell}} F_{\beta}(\beta) \exp \left[-(\ell+2) J\left(\beta ; \beta_{0}, \ell\right)\right]
$$

Equation (23) can be integrated with respect to time yielding

$$
t=H\left(\beta ; a_{0}, \beta_{0}, \ell\right)
$$

ensuring that $H\left(\beta_{0} ; a_{0}, \beta_{0}, \ell\right)=0$.

The actual solution of $\beta$ needs an inversion of Eq. (24),

$$
\beta=H^{-1}\left(t ; a_{0}, \beta_{0}, \ell\right)
$$

Insertion of Eq. (25) into Eq. (22) leads finally to a time evolution $a=a\left(t ; a_{0}, \beta_{0}, \ell\right)$.

\subsection{Asymptotic behaviour of the solution}

The asymptotic value $\beta_{\text {asym }}$ of $\beta$ can be found from Eq. (19) by putting $\dot{\beta}=0$, which enforces a non-linear equation for $\beta_{\text {asym }}$ as

$$
I_{G \beta}\left(\beta_{\text {asym }}\right) I_{Q a a}\left(\beta_{\text {asym }}\right)=I_{G a}\left(\beta_{\text {asym }}\right) I_{Q a \beta}\left(\beta_{\text {asym }}\right) .
$$

If we insert the value $\beta_{\text {asym }}$ into Eq. (16) and take $\dot{\beta}=0$ into account, we find an asymptotic evolution equation for $a$ as 


$$
\dot{a}=\frac{\gamma}{a^{\ell+1} b_{\ell}} \frac{I_{G a}\left(\beta_{\text {asym }}\right)}{I_{Q a a}\left(\beta_{a s y m}\right)} .
$$

This is exactly the evolution equation for $a$, as it was studied in detail by the authors in the recent paper [7] for the case that only $a$ is a time dependent parameter.

\subsection{Determination of the critical radius $R_{c}$}

The critical radius $R_{c}$ is defined in [5], sect. 3 in such a way that $\dot{R}\left(R_{c}\right)=0$ yielding the equation

$$
\dot{a} \int_{R_{c}}^{\infty} \frac{\partial f}{\partial a} d R^{\prime}+\dot{\beta} \int_{R_{c}}^{\infty} \frac{\partial f}{\partial \beta} d R^{\prime}=f \dot{R}, \quad \dot{R}\left(R_{c}\right)=0
$$

Equation (28) can be reformulated with Eqs. (2), (5), (21) and leads to the final relation for

$$
r_{c}=R_{c} / a \text { as }
$$

$$
\frac{I_{G \beta}(\beta) I_{Q a \beta}(\beta)-I_{G a}(\beta) I_{Q \beta \beta}(\beta)}{I_{G \beta}(\beta) I_{Q a a}(\beta)-I_{G a}(\beta) I_{Q a \beta}(\beta)}=\frac{F_{a}(\beta)}{F_{\beta}(\beta)}=\left(\int_{r_{c}}^{\infty} \frac{\partial h}{\partial \beta} d r\right) /\left(3 \int_{r_{c}}^{\infty} h d r-r_{c} h\left(r_{c}, \beta\right)\right) .
$$

Integration per parts is used in the last integral. The solution of Eq. (29) with respect to $r_{c}$ provides the value of the critical radius $R_{c}=\operatorname{ar}_{c}(\beta)$.

\section{Examples}

\subsection{The Kirkaldy-distribution function}

The authors of [7] have discussed the Kirkaldy-distribution function as one of the simplest 2-parameter distributions reading

$$
f_{K}=\frac{h_{K}}{a^{4}}, \quad h_{K}=\frac{15}{\beta^{4}}\left(\frac{r^{2}}{\beta^{2}}-\frac{r^{6}}{\beta^{6}}\right) .
$$

The quantity $\beta$ acts in this case as a so called cut-off dimension-free radius, which was suggested by Kirkaldy to be $32 / 21$ for the coarsening case. One may tend to check whether 
$\beta_{\text {asym }}$ obtains the value $32 / 21$ by using Eq. (26). However, one can show that Eq. (26) is trivially fulfilled for any value of $\beta_{\text {asym }}$. Moreover, also the determinant $D=0$ for any value of $\beta$.

The explanation of both facts can be identified from the distribution function itself, expressed in the variable $R$ as

$$
f_{K}(R, a, \beta)=\frac{15}{a^{4} \beta^{4}}\left(\frac{R^{2}}{a^{2} \beta^{2}}-\frac{R^{6}}{a^{6} \beta^{6}}\right),
$$

which clearly shows that de facto only one parameter, namely $a \beta$, is involved in $f_{K}$, and thus, Kirkaldy's proposal is a one-parameter distribution function.

\subsection{The Weibull-type distribution function}

As dealt with by Streitenberger et al. [3] and discussed in [7] a widely applicable class of distribution functions is a Weibull-type distribution function. The original version of this distribution includes 3 parameters, namely $a, \beta$ and cut-off radius $r_{0}$, which is e.g. 1.5 in the prominent LSW-distribution and 2.0 in the Hillert-distribution, see also [7], sect. 1. For $r_{0} \rightarrow \infty$ and $\beta=0$ we have the classical Rayleigh-distribution function. For $r_{0} \rightarrow \infty$ and $\beta \neq 0$ the distribution function reads

$$
h_{W}=L_{W} r^{\beta+1} \exp \left(-\frac{3 r^{\beta+2}}{\beta+2}\right), \quad L_{W}=\frac{(\beta+2)^{\frac{-3}{\beta+2}} \cdot 3^{\frac{\beta+5}{\beta+2}}}{\Gamma((\beta+5) /(\beta+2))}, \quad L_{W}{ }^{\prime}=\frac{d L_{W}}{d \beta} .
$$

In the current case we do not restrict to a specific value of $\beta$ and let $\beta$ develop starting from an initial value $\beta_{0}$. To calculate the second derivates of $Q$ with respect to $\dot{a}$ and $\dot{\beta}$ we can use exact relations for the integrals

$$
\int_{r}^{\infty} \frac{\partial f_{W}}{\partial a} d r^{\prime}=\frac{L_{W}}{a^{5}} \exp \left(-\frac{3 r^{\beta+2}}{\beta+2}\right)\left(r^{\beta+2}-1\right)
$$




$$
\int_{r}^{\infty} \frac{\partial f_{W}}{\partial \beta} d r^{\prime}=\frac{L_{W}}{a^{4}} \exp \left(\frac{-3 r^{\beta+2}}{\beta+2}\right)\left[\frac{1}{3} \frac{L_{W}^{\prime}(\beta)}{L_{W}(\beta)}+\frac{r^{\beta+2}}{(\beta+2)}\left(\frac{1}{\beta+2}-\ln r\right)\right] .
$$

All further integrals with respect to $Q$ in Eqs. (13c-e) can also be performed analytically e.g. by using MAPLE (http://www.maplesoft.com). But the integration procedure leads to extremely lengthy expressions in variable $\beta$.

The integrals $(13 a, b)$ can also be evaluated analytically and read as

$$
\begin{aligned}
& I_{G a}(\beta)=3^{\frac{1}{\beta+2}}(\beta+2)^{\frac{\beta+1}{\beta+2}} \Gamma\left(\frac{\beta+4}{\beta+2}\right) / \Gamma\left(\frac{\beta+5}{\beta+2}\right), \\
& I_{G \beta}(\beta)=\frac{d}{d \beta} I_{G a}(\beta) .
\end{aligned}
$$

We can calculate $\beta_{\text {asym }}$ from the steady-state condition (26) yielding for the

Growth case: $\beta_{\text {asym }}=0.50703, \quad$ Coarsening case: $\beta_{\text {asym }}=1.86659$.

The functions $F_{a}(\beta), F_{\beta}(\beta)$ in Eqs. (18), (19) can nearly exactly be fitted by quadratic polynomials in the variable $\left(\beta_{\text {asym }}-\beta\right)$ as

Growth case:

$$
\begin{aligned}
& F_{a}(\beta)=0.37519+0.30307\left(\beta_{\text {asym }}-\beta\right)+0.25511\left(\beta_{\text {asym }}-\beta\right)^{2}, \\
& F_{\beta}(\beta)=5.13085\left(\beta_{\text {asym }}-\beta\right)-0.05895\left(\beta_{\text {asym }}-\beta\right)^{2}
\end{aligned}
$$

Coarsening case:

$$
\begin{aligned}
& F_{a}(\beta)=0.16169+0.07278\left(\beta_{\text {asym }}-\beta\right)+0.11919\left(\beta_{\text {asym }}-\beta\right)^{2}, \\
& F_{\beta}(\beta)=7.47000\left(\beta_{\text {asym }}-\beta\right)-0.87085\left(\beta_{\text {asym }}-\beta\right)^{2} .
\end{aligned}
$$

Note that the function $F_{\beta}(\beta)$ is nearly a linear function in $\beta$ and $F_{\beta}\left(\beta_{\text {asym }}\right)=0$. This allows to express $a(\beta)$, Eq. (22), in a quite simple, nearly exact analytical form as following: 


$$
a(\beta)=a_{0}\left(\frac{\beta_{\text {asym }}}{\beta_{\text {asym }}-\beta}\right)^{b} \cdot C(\beta), \quad C(\beta)=1.0+C_{1} \beta+C_{2} \beta^{2},
$$

Growth case: $b=0.07312, \quad C_{1}=0.086, \quad C_{2}=-0.023$;

Coarsening case: $b=0.02164, \quad C_{1}=0.045, C_{2}=-0.009$.

This rather simple equation allows to find via Eq. (23) the equation for $\beta$ as function of a dimension-free time parameter $\tau, \tau=\gamma t /\left(a_{0}^{\ell+2} b_{\ell}\right)$ with $\beta_{0}=0$ as

$$
\int_{0}^{\beta}\left(\frac{\beta_{\text {asym }}}{\beta_{\text {asym }}-\tilde{\beta}}\right)^{b(\ell+2)} \frac{C(\tilde{\beta})^{(\ell+2)}}{F_{\beta}(\tilde{\beta})} d \tilde{\beta}=\tau .
$$

Since the exponent $b(\ell+2)$ is 0.14624 for growth and 0.06492 for coarsening, resp., the integral above has no analytical solution. Of course, the above relation must be inverted to find to a functional relation $\beta=\beta(\tau)$. Insertion of $\beta(\tau)$ into the according Eq. (22) yields finally $a(\tau)$.

Let us study two limiting cases, namely $\beta \rightarrow 0$ (short term solution) and $\beta \rightarrow \beta_{\text {asym }}$ (long term solution). For $\beta \rightarrow 0$ we can use in Eq. (37) the values $C=1$ and $F_{\beta}=F_{\beta}(0)$. Then we find after a short analysis the linear relation

$$
\beta=F_{\beta}(0) \tau
$$

It is well known, that the parameter scaling the radius obeys the parabolic (cubic) law in the steady state of growth (coarsening) as

$$
a^{(\ell+2)}-a_{0}^{(\ell+2)}=\alpha t
$$

with $\alpha$ being a material parameter. Using the dimension-free time parameter $\tau$, one can rewrite ( $\bar{\alpha}$ being a material parameter again) the law as

$$
\frac{a^{(\ell+2)}}{a_{0}^{(\ell+2)}}=1+\bar{\alpha} \tau
$$


Just this form of equation is obtained by linearization of Eq. (36), raised to the power by $(\ell+2)$. Insertion of Eq. (38) yields

$$
\frac{a^{(\ell+2)}}{a_{0}^{(\ell+2)}}=1+(\ell+2)\left(\frac{b F_{\beta}(0)}{\beta_{\text {asym }}}+C_{1}\right) \tau \quad \text { or } \quad a=a_{0}\left[1+(\ell+2)\left(\frac{b F_{\beta}(0)}{\beta_{\text {asym }}}+C_{1}\right) \tau\right]^{\frac{1}{(\ell+2)}}
$$

The direct linearization of Eq. (36) and the use of Eq. (38) provide

$$
a=a_{0}\left(1+\left(\frac{b F_{\beta}(0)}{\beta_{\text {asym }}}+C_{1}\right) \tau\right)
$$

The quantity $b F_{\beta}(0) / \beta_{\text {asym }}$ obtains the value 0.37298 for the growth case and 0.12647 for the coarsening case, resp.

The comparison of the short term and numerical solutions is presented in Figure 1 for both the growth case (Fig. 1 a), c)) and coarsening case (Fig. 1 b), d)). The solution obtained numerically by direct integration of Eqs. (18) and (19) can be considered as an exact one and represents a reference solution. The solution given by Eq. (36) with the use of the values of $\beta$ from the numerical solution provides the solution for $a$ within an accuracy of $1 \%$ for both cases. Using Eqs. (38) and (41), one obtains a very good agreement for $\tau<0.1$. For larger times, however, the values of $a$ differ by a factor of up to 1.26 for the growth case and by a factor of up to 1.58 for the coarsening case. Moreover, no asymptotic behaviour for $\beta$ is obtained. Using Eqs. (38) and (42), one obtains a very good agreement for $\tau<0.1$ and a disagreement for larger times.

From Figure $1 \mathrm{~b}), \mathrm{d})$ it is evident, that the dependence $\beta(\tau)$ can be described quite well by the relation (38) up to the time

$$
\tau^{*}=\beta_{\text {asym }} / F_{\beta}(0)
$$

and then by keeping

$$
\beta=\beta_{\text {asym }} \text { for } \tau>\tau^{*} \text {. }
$$


The short term solution for $a$ is given by Eq. (41) for $\tau \leq \tau^{*}$ and $a$ reaches the value

$$
a^{*}=a_{0}\left[1+(\ell+2)\left(b+\frac{C_{1} \beta_{a s y m}}{F_{\beta}(0)}\right)\right]^{\frac{1}{(\ell+2)}}
$$

for $\tau=\tau^{*}$. For $\tau>\tau^{*}$ one can insert $\beta=\beta_{\text {asym }}$ into Eq. (18) and its direct integration provides the long term solution:

$$
a^{(\ell+2)}-a^{*(\ell+2)}=(\ell+2) \frac{\gamma}{b_{\ell}} t F_{a}\left(\beta_{\text {asym }}\right)=a_{0}^{\ell+2}(\ell+2) F_{a}\left(\beta_{\text {asym }}\right)\left(\tau-\tau^{*}\right), \quad \tau>\tau^{*} .
$$

Again the parabolic (cubic) law can be recognized for $\tau>\tau^{*}$. The solution $\beta(\tau)$ and $a(\tau)$ given by the combination of the short term solution (Eqs. (38) and (41)) for $\tau \leq \tau^{*}$ and the long term solution (Eqs. (44) and (46)) for $\tau>\tau^{*}$ is presented by triangles in Figure 1a,b. The deviation of $a(\tau)$ from the reference solution is less then $1 \%$ in the whole interval $0 \leq \tau<\infty$

The evolution of $h_{W}(\tau)$ given by Eq. (32) for different values of $\tau$ is presented for both the growth and coarsening case in figure 2. The function $h_{W}(\tau)$ stops to evolve for the normalized time $\tau \geq 1$, which corresponds to the values of $\tau$, for which $\beta \approx \beta_{\text {asym }}$. The evolution of distribution function $f_{W}(\tau)$ given by Eqs. (2) and (32) for different values of $\tau$ for both the growth and coarsening case is presented in figure 3.

The critical radius $R_{c}$ is investigated according to sect. 3.3. For the left hand side of Eq. (29) we use the fits from Eqs. (35a-d). The integrals on the right hand side of Eq. (29) can be found analytically. It can easily be shown that for $\beta=\beta_{\text {asym }}$ the critical radius is $a$. The numerical solution of Eq. (29) both for the growth and coarsening case are shown in Fig. 4. It is interesting to note that $r_{c}=R_{c} / a$ deviates not significantly from 1 in the interval 
$0 \leq \beta \leq 2 \beta_{\text {asym }}$. The reason for running $\beta$ beyond $\beta_{\text {asym }}$ lies in the fact that in general one may start with any $\beta$-value.

Finally we list the kinetic equation as evolution equation for $\dot{R}$ according to the first relation (28) using the exact integrals (33a), (33b). After some analysis we find with Eqs.

$$
R^{\ell+1} \dot{R}=\frac{\gamma}{b_{\ell}} r^{\ell}\left\{F_{a}(\beta)\left(r^{2}-r^{-\beta}\right)+\frac{F_{\beta}(\beta)}{(\beta+2)}\left[\left(\frac{1}{\beta+2}-\ln r\right) r^{2}+\frac{1}{3} \frac{L_{W}{ }^{\prime}}{L_{W}} r^{-\beta}\right]\right\} .
$$

The first part of the above kinetic expression agrees with the mathematical structure of the kinetics of the one-parameter Weibull-type distribution in [7], Eqs. (20), (21), however, with a fixed value of $\beta$ there. The second contribution to kinetics refers to the term $\dot{\beta}$ and approaches zero, when $\beta \rightarrow \beta_{\text {asym }}$. The most important feature of this kinetics is that $\beta$ is now a function evolving with the time.

\section{Discussion and outlook}

A non-steady object-radius distribution function is described by two parameters. The first parameter, $a$, scales the object radius $R$ and can be considered as the "representative" object radius. The second parameter, $\beta$, is dimension-free and characterizes the shape of the radius distribution function.

One can show that

- $\quad \beta$ is now not a somewhat arbitrary parameter but tends to a distinct value $\beta_{\text {asym }}$, which differs for the growth and coarsening case (depends on controlling process) and can be determined from the PMD,

- an explicit evolution equation can be given for $\dot{a}(a, \beta)$ and $\dot{\beta}(a, \beta)$, so finally the evolution of $a$ as well as $\beta$ can be calculated by numerical integration in time, 
- an explicit functional relation can be determined for $a(t)$ and $\beta(t)$.

This allows now to follow actual distributions during their evolution with a dimensionfree time parameter $\tau$ being either $\left(\gamma M / a_{0}^{2}\right) t$ in the growth case or $\left(\gamma /\left(\varepsilon a_{0}^{3}\right)\right) t$ in the coarsening case.

The study of a Weibull-type distribution function gives a clear evidence that the evolution of the parameter scaling the object radius $R$ represented by $a(\tau)$, follows a "parabolic" or "cubic" regime with a changing parameter $\beta(\tau)$.

As a future work the following two tasks seem to be worth while for solving:

- Treatment of precipitation controlled by bulk diffusion and additionally by interface reaction. In this case the dissipation consists of two terms due to bulk diffusion $\square R^{3} \dot{R}^{2}$ and due to interface reaction (analogy to the grain boundary migration) $\square R^{2} \dot{R}^{2}$. In that case the features of grain growth and precipitate coarsening are coupled. Growth mechanism dominates for fine precipitate structure and mechanism of coarsening dominates for coarse precipitate structure. As the relations of the controlling mechanisms change during the system evolution, one can expect that the $\beta$ parameter approaches its asymptotic value very slowly.

- A third parameter may be brought into play, e.g. the so-called cut-off parameter $r_{0}$, restricting the distribution $f(R)$ to the interval $0 \leq r \leq r_{0}=R_{0} / a$. The cut-off parameter $r_{0}$ is set to 2 in the Hillert-distribution or 1.5 in the LSW-distribution and is discussed by Zöllner and Streitenberger [3] for the Weibull-type distribution function. In this case the matrix of the set of linear equations for $\dot{a}, \dot{\beta}$ and $\dot{r}_{0}$ is of dimension 3 with the unchanged original $2 \times 2$ submatrix corresponding to $\dot{a}$ and $\dot{\beta}$ if $r_{0} \rightarrow \infty$. The solution of 


\section{Summary}

The content of the paper can be summarized into the following items:

- In the simplest case the non-steady-state evolution of the object radii distribution can be described by a two-parameter function. The first parameter $a(t)$ can be used for the scaling of the object radius $R$ and the second one, $\beta(t)$, influences the shape of the distribution function for the normalized coordinate $r=R / a$.

- The evolution equations $\dot{a}=\dot{a}(a, \beta)$ and $\dot{\beta}=\dot{\beta}(a, \beta)$ can be derived by application of principle of maximum dissipation (PMD) for an arbitrary two-parametric distribution function and their semi-analytical solution can be developed.

- The general evolution equations are applied to Kirkaldy and Weibull-type two-parameter distribution functions. The Kirkaldy distribution function is identified having two dependent parameters, and the general analysis fails. On the other hand, the Weibull-type distribution function is analyzed in detail.

- The evolution equations for parameters of the Weibull-type distribution function are integrated numerically with respect to time. This solution is considered as the exact reference solution. Several simple approximate analytical solutions are presented. A 
combination of a short term and a long term solution seems to be very successful and agrees with the reference solution within one percent for $a(t)$ and, except to a short transient period, almost exactly also for $\beta(t)$.

\section{Acknowledgement}

Financial support by Materials Center Leoben Forschung GmbH (project SP19) and by Research plan of Institute of Physics of Materials (project CEZ:AV0Z20410507) is gratefully acknowledged.

\section{References}

[1] Y. Suwa, Y. Saito, H. Onodera, Acta mater. 55 (2007) p. 6881.

[2] Y. Suwa, Y. Saito, H. Onodera, Materials Transactions 49 (2008) p. 704.

[3] D. Zöllner, P. Streitenberger, Scripta mater. 54 (2006) p. 1697.

[4] F.D. Fischer, J. Svoboda, P. Fratzl, Philos. Mag. 83 (2003) p. 1075.

[5] J. Svoboda, F.D. Fischer, Acta mater. 55 (2007) p. 4467.

[6] F.J. Humphreys, M. Hatherly, Recrystallization and Related Annealing Phenomena, Sect., 9.25, Pergamon, Elsevier, Oxford, 1995.

[7] F.D. Fischer, J. Svoboda, E. Gamsjäger, E.R. Oberaigner, Acta mater. 56 (2008) p. 5395.

[8] M. Groeber, S. Ghosh, M.D. Uchic, D.M. Dimiduk, Acta mater. 56 (2008) p. 1257.

[9] M. Groeber, S. Ghosh, M.D. Uchic, D.M. Dimiduk, Acta mater. 56 (2008) p.1274.

[10] L.A. Barrales Mora, G. Gottstein, L.S. Shvindlerman, Acta mater. 56 (2008) p.5915.

[11] J. Jeppsson, J. Agren, M. Hillert, Acta mater. 56 (2008) p. 5188.

\section{Figure captions}


Figure 1: Comparison of different solutions $a(\tau)$ and $\beta(\tau)$ for growth case $-\mathrm{a})$ and c) and coarsening case $-b$ ) and d).

Figure 2: Evolution of $h_{W}(\tau)$ given by Eq. (32) for different values of $\tau$. a) growth case, b) coarsening case.

Figure 3: Evolution of distribution function $f_{W}(\tau)$ given by Eqs. (2) and (32) for different values of $\tau$. a) growth case, b) coarsening case.

Figure 4: Dependence of normalized critical radius $r_{c}$ on parameter $\beta$ being a solution of Eq. (29) with $h=h_{W}$. a) growth case, b) coarsening case. 


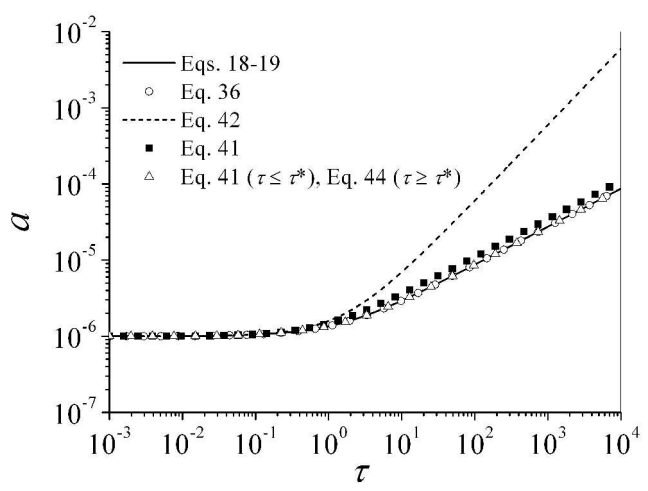

a)

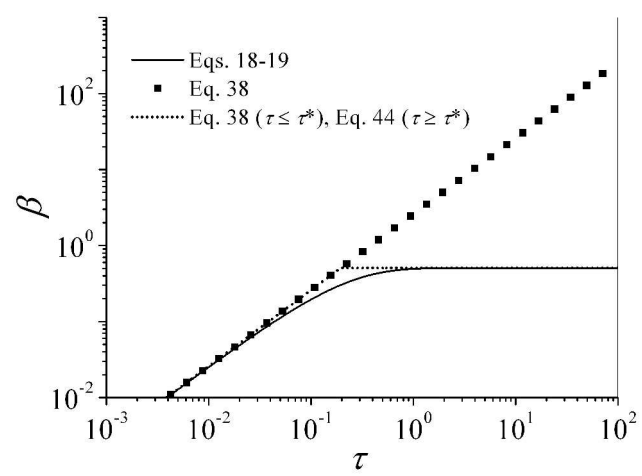

c)

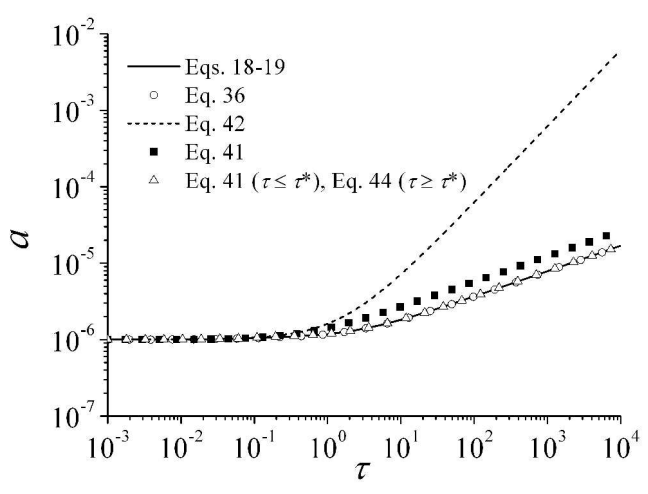

b)

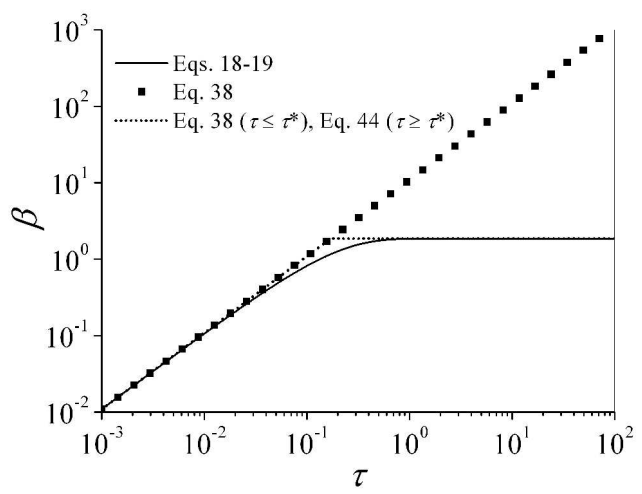

d)

Figure 1: Comparison of different solutions $a(\tau)$ and $\beta(\tau)$ for growth case $-\mathrm{a})$ and c) and coarsening case $-b$ ) and d).

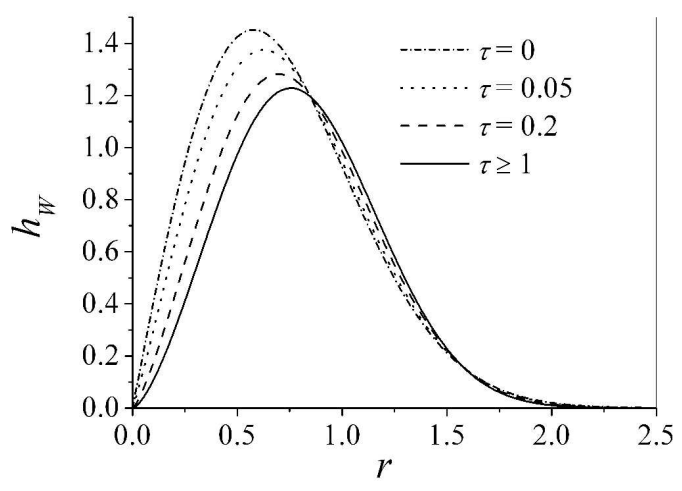

a)

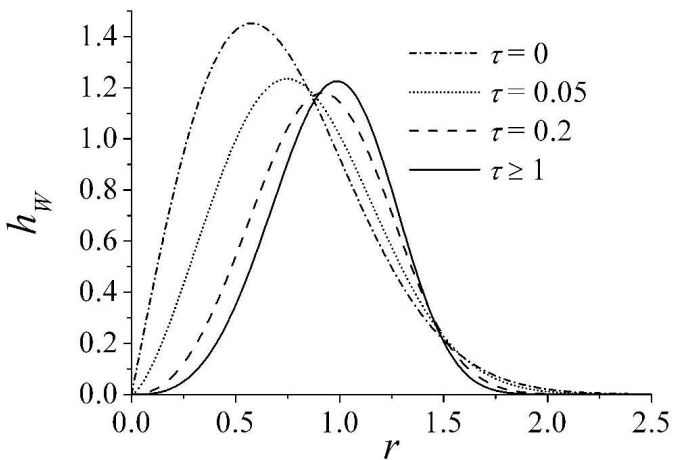

b)

Figure 2: Evolution of $h_{W}(\tau)$ given by Eq. (32) for different values of $\tau$. a) growth case, b) coarsening case. 


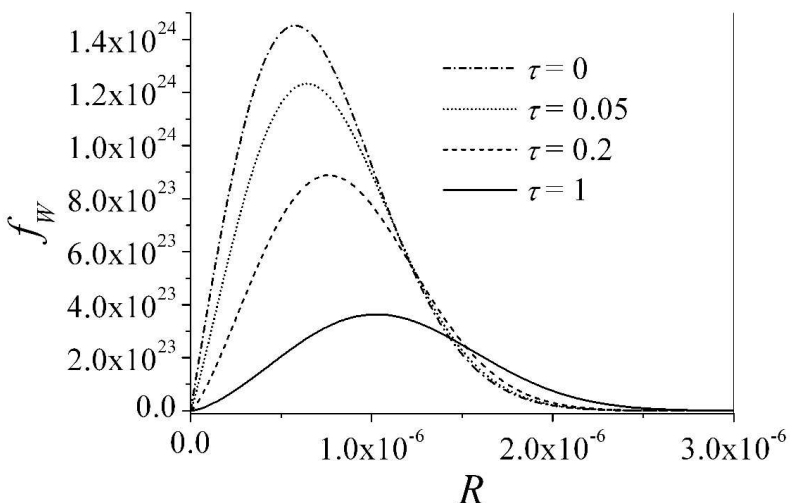

a)

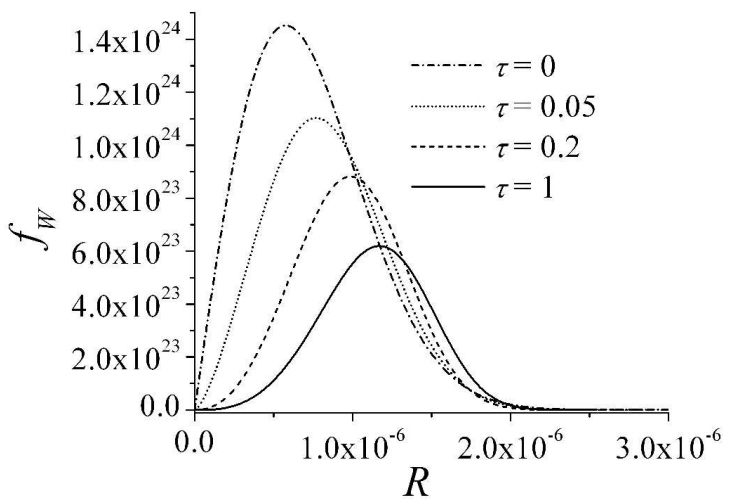

b)

Figure 3: Evolution of distribution function $f_{W}(\tau)$ given by Eqs. (2) and (32) for different values of $\tau$. a) growth case, b) coarsening case.

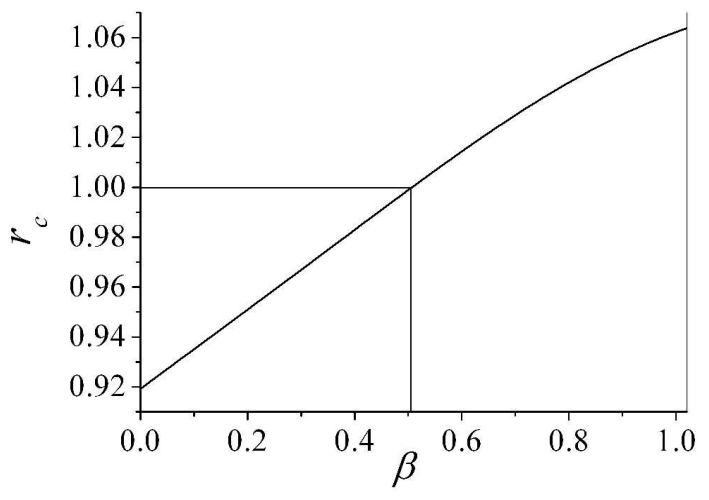

a)

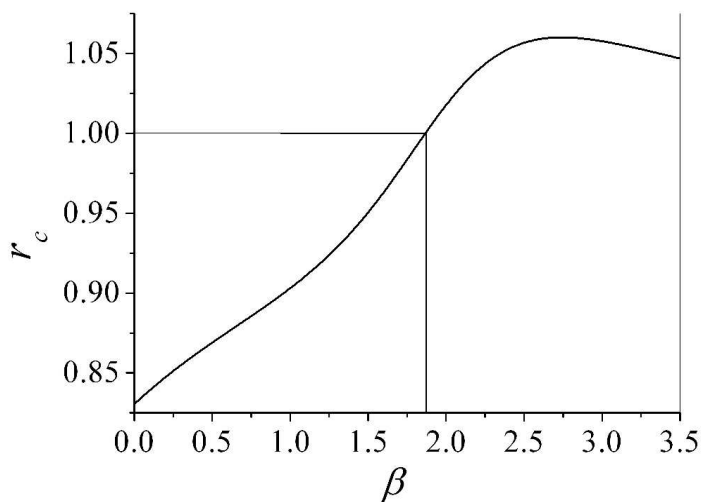

b)

Figure 4: Dependence of normalized critical radius $r_{c}$ on parameter $\beta$ being a solution of Eq. (29) with $h=h_{W}$. a) growth case, b) coarsening case. 